Comment on Guo, Li, Wang, Cai and Duncan

\author{
John Levi Martin \\ University of Chicago \\ 1126 E $59^{\text {th }}$ Street \\ Chicago IL 60637 \\ jlmartin@uchicago.edu
}

Keywords: Gene-environment, alcohol, residual variation, misspecification 
Comment on Guo, Li, Wang, Cai and Duncan

\begin{abstract}
Guo, Li, Wang, Cai and Duncan (2015) recently claimed to have provided evidence for a general theory of gene-environment interaction. The theory holds that those who are labelled as having high or low genetic propensity to alcohol use will be unresponsive to environmental factors that predict binge-drinking among those of moderate propensity. They actually demonstrate evidence against their theory, but do not seem to have understood this.
\end{abstract}




\section{Comment on Guo, Li, Wang, Cai and Duncan}

Guo, Li, Wang, Cai and Duncan's recent (2015) paper in the AJS (henceforward, GLWCD) purports to be about gene-environment interactions, and to confirm a general hypothesis about this relation. However, it suffers from an elementary error of logic, and it actually has demonstrated evidence against the main claim...despite the fact that this claim is a plausible one for any two sets of predictors.

The key argument in the genetic swing theory is that "individuals with a medium genetic propensity are more likely to be swayed by an adverse environmental influence to develop a risk behavior than individuals at the extreme with a low and high propensity" (921). This is a true statement of the theory's implications, although GLWCD's actual approach to testing this claim is not to compare effects to one another but to compare them severally to zero. (We return to this point briefly below, and consider the conclusions if we took an analytic approach more in keeping with GLWCD's description here.)

"In the current study," continue Guo et al, "swing theory predicts that individuals with a medium propensity for binge drinking are more likely to be influenced by a binge-drinking roommate than are individuals with a low or high propensity. Individuals with a very low genetic propensity tend not [to] be affected because they are inherently disinterested in alcohol use. Individuals with a high genetic propensity tend not [to] be influenced by peers because of already developed drinking habits." This is only partially correct. More accurate would be the following: "In the current study, swing theory predicts that individuals with a medium propensity for binge drinking are more likely to be influenced by any potential environmental determinants than are individuals with a low or high propensity." 
Even more important, the logic of the swing theory is that if some predictor has an effect, then it will have its effect in the medium, and not the extreme, propensity tiers. No particular predictor must have an effect. Therefore, swing theory makes stronger predictions for the extreme propensity categories (where there cannot be any effect) than for the middle (where there may or may not be such an effect).

This is consequential because of the way that choose to examine their data. Although the verbal description of the swing theory here refers to the comparison of magnitudes ("more likely"), the methods used by GLWCD involve successive tests of the null hypothesis across three subsets formed by partitioning the sample by level of what is termed genetic propensity. If we denote these three subsets $L, M$ and $H$, standing for low, medium and high propensity, then, for the $k^{\text {th }}$ predictor, they estimate three slopes, $b^{L}{ }_{k}, b^{M}{ }_{k}$, and $b^{H}{ }_{k}$. Because the swing theory does not require that any particular predictor have an effect, but only that if it does, it does not in the extreme propensity tiers, this theory holds that for any $k, b^{L}{ }_{k} \approx b^{H}{ }_{k} \approx 0$.

Once we correctly assess the implications of their theory, we find that GLWCD's results strongly militate against their claims. Beginning with the results from the ROOM study, we find that indeed, for the three dependent variables, the roommate-drinking-in-high-school variable is larger in the MEDIUM than in the HIGH or LOW propensity groups, and in two it is significant at $p<.05$. But roommate's family income, which is also an experimental treatment, is larger in the HIGH propensity category than in the other two, and is statistically significant at $\mathrm{p}<.05$ for two of the dependent variables. Further, the causal nature of this pathway is quite plausible, as those of us who were fortunate (or unfortunate) enough to be placed with a wealthier roommate in college know. The roommate's income (or, more accurately, his/her possession of some sort of allowance) may have a direct effect on the focal subject's drinking if it leads the roommate to 
treat or lend money to the focal student, enabling binge drinking, or if it increases the popularity of the focal students, leading to invitations to events at which binge drinking takes place. Thus those of high propensity seem, contrary to hypothesis, to be swingable. If they are more likely to binge drink when a roommate is paying the tab (say), it seems doubtful that they are so boundand-determined to drink that they are immune to peer effects, as held by GLWCD.

Those of LOW propensity also appear to be swingable, though in this case, it is due to the non-randomly allocated variable of church attendance, which is significant for two of the dependent variables, and larger in magnitude than the coefficient for the MEDIUMs in one. It is of course possible that an unmeasured genetic propensity here has affected both their tendency to go to church and to avoid binge drinking. For this reason, these results may be considered less weighty than those derived from the randomized treatment, though we might be reassured by GLWCD's catholic search for any genetic predictors of alcohol use that few such propensities have escaped being measured. Further, GLWCD argue that the differences in effect between randomly allocated and non-randomly allocated treatments in terms of their interaction with genes may not be as large as feared (946). But in any case, there is at least as much evidence in Table 4 against GLWCD's hypothesis as there is for it.

When it comes to the ADD-HEALTH data (Table 5), we have no experimental treatments; all the variables are on the same footing. (While we might not use the coefficients of some temporally early variables as estimates of their causal effects, given that potential posttreatment confounders are present in the model, we can use their significance to reject the null hypothesis of no direct effect.) First, it should be noted that the data here are displayed in a misleading format. Because of the varying counts in the three categories, effects that are not statistically significant in one of the extreme propensity groupings may be significant in the 
MEDIUM grouping. Further, tests of significant difference across the categories are not made.

Thus while we can accept that the .72 coefficient for MEDIUMs using the 27 SNP genetic measure is significantly different from zero for the 827 youths in this category, while the coefficient of .65 for the 303 in the HIGH category is not, it would be quite rash to assume that this was an indication that the MEDIUMs were more susceptible than the HIGHs. Indeed, the estimated coefficient of the same effect for the HIGHs using the 5 SNP measure (.71) is larger in magnitude than that for the MEDIUMs (.55), but because of the larger sampler size for the latter, it is the smaller coefficient that is graced with a symbol indicating marginal statistical significance. $^{1}$

In any case, when we peruse the other environmental factors taken into account in this table, we find that household size — a key aspect of the environment for youth —is significant for the HIGH group over the past year, and not the MEDIUM or LOW, while having two biological parents present is more strongly associated with binge drinking (and significantly so, using the 27 SNP measure) for those with LOW propensity when it comes to the past two weeks, but not for the HIGHs or MEDIUMS. Indeed, if we simply survey all coefficients for Add Health in Table 5, and choose the one with the largest absolute value (p-values being misleading given the unequal sizes), we find the following: for the dependent variable for binge drinking in the past

\footnotetext{
1. While the choice of separate comparisons of coefficients to zero within tiers is just as defensible as are tests of comparison of the coefficients across the tiers - there is no perfect solution to the problem that differences of statistical significance are not transitive - GLWCD's choice to suppress standard errors and instead report p-values makes it somewhat difficult for a reader to check whether the comparisons being made are statistically significant. Reverse-engineering the standard errors and using a simple two-tailed, two-sample test for all the $3 \times 2+2 \times 2 \times 2=14$ tests that would be conducted across Tables 4 and 5 finds that only one is statistically significant $\mathrm{p}<.05$ (this is the difference between medium and low propensity groups for the past two weeks dependent variable using the 5 SNP method). That is around what one would expect given so many tests if there was no true relation at all. These results are found in Appendix A .
} 
two weeks, $7.5^{2}$ coefficients are largest in the low propensity category, 8.5 for the medium propensity category, and 16 for the high propensity category (the numbers are 6.5, 6.5 and 15 if we omit the arguably genetic cognitive score). For the past year dependent variable, the numbers are 8,5 and 19 respectively $\left(8,3\right.$, and 17 if we omit cognitive score). ${ }^{3}$

Finally, the reader should note that Tables 6 and 7, assuming what is to be proven, make the above facts difficult to see, by constraining the parameters for environmental effects not of interest to GLWCD to be the same across all genetic categories, but not doing so for the variable which supports their claim, namely, peer influence. ${ }^{4}$ Overall, to summarize, the results are not consistent with a theory in which those of medium genetic propensity are uniquely susceptible to environmental determinants.

GLWCD's paper purports to be about gene-environment interactions. This is not in keeping with the conventional use of this notion, which refers to specific alleles being activated or being determinative in some environments and not others. Such an examination might, for example, be compatible with investigating whether those with high genetic propensity only binge-drink when they are in some environments as opposed to others. Instead, this paper is about a very straightforward statistical issue — how much residual variance is available for a second predictor after a first one has had a crack at the data. The answer actually has nothing to do with the characteristic of which predictors we use. In general, if we have a de facto bounded

2. The .5 's come from a tie, given the 3 digits of significance reported.

3. If we conduct the same test for Table 4, we again find that they tend to be larger in absolute value for the high propensity category; for the three dependent variables in question, the corresponding numbers for the number of low, medium and high maxima are $(4,5,8),(2,4,11)$ and $(3,4.5,9.5)$ respectively, where the .5 comes from a tie.

4. The equations therefore appear to be misleadingly described. The note to the table reads: "Each column presents the coefficients from a single regression model." Were that so, the equality of the control coefficients across tiers would be a remarkable coincidence. 
dependent variable and we partition our data sets according to the levels of a first strong predictor, we expect other predictors to have maximal explanatory power in the middle of the range of the first predictor, and none at the extremes..$^{5}$ That GLWCD did not find this supports what is the current consensus in the social and behavioral sciences - that genetic variation in itself, while a strong predictor for many health-related outcomes, is, so far as we can tell, a very weak predictor for any sort of measurable social behavior.

The reader may be tempted to respond that while GLWCD's claims were incorrectly supported, perhaps we do see evidence of a gene-environment interaction precisely where they believed it should not be, namely, the anomalous (to their claims) significance of predictors among those with HIGH propensity. However, GLWCD's models were not properly specified to allow us to conduct such an examination. When it comes to behavioral genetics, the most difficult task is to disentangle two correlated classes of variables-genotype (call this $z$ ), on the one hand, and (possibly socially ascribed aspects of) biodescent (call this $w$ ), on the other, given that a correlation between $w$ and $z$. If we are interested in the effects of these on some $y$, and suspect that either may interact with some predictor $x$, a conventional approach would be to fit

$$
\hat{y}=b_{x} x+b_{z} z+b_{w} w+b_{x z} x z+b_{x w} x w .
$$

Fitting the model that constrains $b_{x w}=0$ would not be a proper test, for if in the true model, $b_{x w}>0$ and $b_{x z}=0$, omitting the former would lead to an incorrect estimate of $b_{x z}>0$. GLWCD's tactic of splitting the sample into tiers by $z$, and then controlling for $x$, is equivalent to forcing this constraint of $b_{x w}=0 .^{6}$

5. If the reader doubts this, please see Appendix B. GLWCD's measures, while converted to monthly rates, originally come from bounded variables.

6. If the reader doubts this, please see Appendix C. 
Further, there is a clear problem in using such interactions. It certainly makes sense that, if genes are relevant for behavioral outcomes, we would expect gene-environment interactions, since most such outcomes are complex interactions of many different players in addition to the focal subject (e.g., the recording of an arrest, or even participation at a binge drinking episode, requires cooperation). But, it is worth reminding ourselves that because GLWCD took the worrisome tactic of using stepwise regression to compose as a purported measure of genetic propensity — that is, it is simply "whatever genes we have that predict drinking in this sample" it would be likely for it to in fact predict drinking somewhere, even if only by chance. ${ }^{7}$ Indeed, the high rate of non-reproducible findings in the genexenvironment literature is well known (see, recently, Perry 2016: 1656, 1661). Given the difficulty of disentangling biodescent and genes, especially in diverse samples, pursuing interactions in the absence of theory radically increases the already well documented tendency of such research to reach false positives (see, e.g., Chabris et al. 2012).

7. GLWCD employed a version of the Benjamini and Hochberg (1995) technique for dealing with multiple simultaneous tests; presumably they use the version developed for correlated tests. However, they do not merely attempt to determine whether the evidence favors rejecting one or more null hypotheses, but go on to create an propensity score by adding weighted versions of the significant predictors. This weighting, so far as I can see, is not corrected for by the $\mathrm{BH}$ procedure, and so there is no reason to be confident that it will not parameterize sampling error. The initial selection via $\mathrm{BH}$ is, in other words, only used to gather the strongest predictors, and not to test the claim that there is genetic causation of binge drinking. This is done via conventional statistics tests of the propensity measure. Further, it does not seem likely that a correction that is made for the sample as a whole is what one would want if then one is going to split the sample and come to conclusions based on these sub-samples. 


\section{References}

Benjamini, Yoav and Yosef Hochberg. 1995. "Controlling the False Discovery Rate: A Practical and Powerful Approach to Multiple Testing." Journal of the Royal Statistical Society B 57: 289-300.

Chabris, Christopher F. et al. 2012. "Most Reported Genetic Associations with General Intelligence and Probably False Positives.” Psychological Science 23:1314-1323.

Guo, Guang, Yi Li, Hongyu Wang, Tianji Cai and Greg J. Duncan. 2015. "Peer Influence, Genetic Propensity and Binge Drinking: A Natural Experiment and a Replication.” American Journal of Sociology 121: 914-954.

Perry, Brea L. 2016. "Gendering Genetics: Biological Contingencies in the Protective Effects of Social Integration for Men and Women.” American Journal of Sociology 121:16551696. 
Appendix A: Re-casting the tests as comparisons between coefficients.

GLWCD's first verbal presentation of their theory involved statements of the comparison across coefficients - that those in medium propensity groups should be more likely to be swayed by influence than those in low or high propensity groups. This would imply a comparison of coefficients, a comparison which GLWCD did not present. Their decision is not indefensible nor uncommon-because of our imprecision, we might be confident that the coefficient in one group is not zero, be unable to make the same rejection of the null hypothesis for a second group, yet also be unable to reject the possibility that the two are different. But while GLWCD's choice of analysis is common, less common is their decision not to print standard errors, but only p-values, making the non-significance of the comparison of coefficients harder for the reader to uncover. But, given a p-value $(p)$, we can recover the standard error $(s)$ of the coefficient $(b)$ by first constructing the inverse of the cumulative normal distribution $f$ for $p\left(F(p)=f^{1}(p)\right)$, setting this equal to $b / s$, and solving for $s$. With this, we can then construct the standard error for the test that $b_{1}=b_{2}$ for two slopes from different samples with standard errors $s_{1}$ and $s_{2}$ respectively, simply by dividing $b_{1}-b_{2}$ by $s^{*}=\operatorname{sqrt}\left(s_{1}{ }^{2}+s_{2}{ }^{2}\right)$ and examining how much of the distribution lies beyond this as a standardized value.

Note that a positive coefficient that is statistically significantly different from zero may not be statistically significantly different from another coefficient that is less than zero. This is because the imprecision of the estimate of the second coefficient means that we are not confident that it is, in fact, less than zero, while we treat zero as fixed with absolute precision. The results below show two-tailed tests for every pair of comparisons for all of GLWCD's models in Tables 4 and 5. For each table, for each dependent variable, and, where relevant, for each measure of genetic propensity, I first compare the MEDIUM coefficient to the LOW, and then to the HIGH. The first column is the reported coefficient, the second, the reported p-value of this coefficient, the second, the corresponding $Z$ score (the inverse of the cumulative distribution function), and the fourth, the standard error of the coefficient. The fifth column, labeled "t $t$ est]-value," is the difference in coefficients divided by the joint standard error, and the sixth column is the significance of this difference. The one significant result is bolded. The table of results begins on the next page; Table A-1 contains the results for GLWCD's Table 4, and Table A-2 contains the results for GLWCD's Table 5. 
Table A-1: Significance tests of coefficients, GLWCD's Table 4

\begin{tabular}{lrrrrrr} 
& \multicolumn{2}{c}{ Table 4} & \multicolumn{2}{c}{ DVI } & & \\
Medium & Coefficient & $\mathrm{p}$-val & $\mathrm{Z}=\mathrm{F}(\mathrm{p})$ & $\mathrm{SE}$ & $\mathrm{t}$ - value & $\mathrm{p}$-val \\
Low & 0.95 & 0.02 & 2.31 & 0.41 & & \\
Difference & 0.42 & 0.43 & 0.79 & 0.53 & & \\
& 0.54 & & & 0.67 & 0.80 & 0.42 \\
& & & & & & \\
Medium & Coefficient & $\mathrm{p}$-val & $\mathrm{Z}=\mathrm{F}(\mathrm{p})$ & $\mathrm{SE}$ & $\mathrm{t}$ - value & $\mathrm{p}$-val \\
High & 0.95 & 0.02 & 2.31 & 0.41 & & \\
Difference & -0.30 & 0.77 & 0.29 & -1.02 & & \\
& 1.25 & & & 1.10 & 1.14 & 0.26 \\
& & & & & & \\
Medium & & Table 4 & & DV2 & & \\
Low & 0.73 & 0.07 & 1.82 & 0.40 & & \\
Difference & 0.27 & 0.63 & 0.48 & 0.56 & & \\
& 0.46 & & & 0.69 & 0.67 & 0.50 \\
& & & & & & \\
Medium & Coefficient & $\mathrm{p}$-val & $\mathrm{Z}=\mathrm{F}(\mathrm{p})$ & $\mathrm{SE}$ & $\mathrm{t}-\mathrm{v}$-value & $\mathrm{p}$-val \\
High & 0.73 & 0.07 & 1.82 & 0.40 & & \\
Difference & -0.51 & 0.51 & 0.66 & -0.78 & & \\
& 1.25 & & & 0.88 & 1.43 & 0.15
\end{tabular}

Table $4 \quad$ DV3

$\begin{array}{lrrrrrr} & \text { Coefficient } & \text { p-val } & \mathrm{Z}=\mathrm{F}(\mathrm{p}) & \mathrm{SE} & \mathrm{t} \text {-value } & \mathrm{p} \text {-val } \\ \text { Medium } & 0.88 & 0.02 & 2.29 & 0.39 & & \\ \text { Low } & -0.54 & 0.40 & 0.84 & -0.64 & & \\ \text { Difference } & 1.42 & & & 0.75 & 1.90 & 0.06\end{array}$

$\begin{array}{lrrrrrr} & \text { Coefficient } & \mathrm{p} \text {-val } & \mathrm{Z}=\mathrm{F}(\mathrm{p}) & \mathrm{SE} & \mathrm{t}-\text { value } & \mathrm{p} \text {-val } \\ \text { Medium } & 0.88 & 0.02 & 2.29 & 0.39 & & \\ \text { High } & -0.63 & 0.42 & 0.81 & -0.78 & & \\ \text { Difference } & 1.51 & & & 0.87 & 1.74 & 0.08\end{array}$


Table A-2: Significance tests of coefficients, GLWCD's Table 5

\begin{tabular}{|c|c|c|c|c|c|c|}
\hline & & Table 5 & $5 S N P$ & $D V 1$ & & \\
\hline & Coefficient & P-val & $\mathrm{Z}=\mathrm{F}(\mathrm{p})$ & SE & $\mathrm{t}$ - value & p-val \\
\hline Medium & 1.10 & 0.00 & 3.39 & 0.32 & & \\
\hline Low & -0.12 & 0.75 & 0.32 & -0.38 & & \\
\hline \multirow[t]{2}{*}{ Difference } & 1.22 & & & 0.50 & 2.45 & 0.01 \\
\hline & Coefficient & P-val & $\mathrm{Z}=\mathrm{F}(\mathrm{p})$ & SE & $\mathrm{t}$ - value & p-val \\
\hline Medium & 1.10 & 0.00 & 3.39 & 0.32 & & \\
\hline High & 0.41 & 0.30 & 1.04 & 0.40 & & \\
\hline \multirow[t]{3}{*}{ Difference } & 0.69 & & & 0.51 & 1.35 & 0.18 \\
\hline & & Table 5 & $5 S N P$ & $D V 2$ & & \\
\hline & Coefficient & P-val & $\mathrm{Z}=\mathrm{F}(\mathrm{p})$ & SE & $\mathrm{t}$ - value & p-val \\
\hline Medium & 0.55 & 0.06 & 1.89 & 0.29 & & \\
\hline Low & 0.21 & 0.55 & 0.60 & 0.35 & & \\
\hline \multirow[t]{2}{*}{ Difference } & 0.34 & & & 0.46 & 0.75 & 0.46 \\
\hline & Coefficient & P-val & $\mathrm{Z}=\mathrm{F}(\mathrm{p})$ & SE & $\mathrm{t}$ - value & p-val \\
\hline Medium & 0.55 & 0.06 & 1.89 & 0.29 & & \\
\hline High & 0.71 & 0.13 & 1.51 & 0.47 & & \\
\hline Difference & 0.16 & & & 0.55 & 0.29 & 0.77 \\
\hline
\end{tabular}

Table $5 \quad 27$ SNP DVI

Coefficient $\quad \mathrm{P}$-val $\mathrm{Z}=\mathrm{F}(\mathrm{p}) \quad \mathrm{SE} \quad \mathrm{t}$ - value $\quad \mathrm{p}$-val

$\begin{array}{lllll}\text { Medium } & 0.76 & 0.02 & 2.39 & 0.32\end{array}$

$\begin{array}{lllll}\text { Low } & 0.52 & 0.07 & 1.79 & 0.29\end{array}$

$\begin{array}{lllll}\text { Dif } & 0.24 & 0.43 & 0.56 & 0.58\end{array}$

$\begin{array}{lrrrrrr} & \text { Coefficient } & \text { P-val } & \mathrm{Z}=\mathrm{F}(\mathrm{p}) & \mathrm{SE} & \mathrm{t}-\text { value } & \mathrm{p} \text {-val } \\ \text { Medium } & 0.76 & 0.02 & 2.39 & 0.32 & & \\ \text { High } & 0.35 & 0.53 & 0.63 & 0.56 & & \\ \text { Dif } & 0.41 & & & 0.64 & 0.64 & 0.52\end{array}$

\begin{tabular}{|c|c|c|c|c|c|c|}
\hline & & able 5 & $27 S N P$ & $D V 2$ & & \\
\hline & Coefficient & P-val & $\mathrm{Z}=\mathrm{F}(\mathrm{p})$ & SE & $\mathrm{t}$ - value & $\mathrm{p}$-val \\
\hline Medium & 0.72 & 0.02 & 2.43 & 0.30 & & \\
\hline Low & 0.06 & 0.84 & 0.20 & 0.30 & & \\
\hline Dif & 0.66 & & & 0.42 & 1.57 & 0.12 \\
\hline
\end{tabular}

Continued.... 


$\begin{array}{lrrrrrr} & \text { Coefficient } & \text { P-val } & \mathrm{Z}=\mathrm{F}(\mathrm{p}) & \mathrm{SE} & \mathrm{t} \text { - value } & \mathrm{p} \text {-val } \\ \text { Medium } & 0.72 & 0.02 & 2.43 & 0.30 & & \\ \text { High } & 0.65 & 0.24 & 1.17 & 0.55 & & \\ \text { Dif } & 0.07 & & & 0.63 & 0.11 & 0.91\end{array}$


Appendix B: For those who doubt the claim that any strong predictor will produce the effects claimed for "swing theory."

I noted that if we have a de facto bounded dependent variable and we partition our data sets according to the levels of a strong predictor, we expect other predictors to have maximal explanatory power in the middle of the range of the strong predictor. It should be emphasized that this does not necessarily hold for unbounded variables, and that even for loglinear type models (such as the logistic regression) for bounded variables, this is not a mathematical necessity (since such models are technically variance-indifferent). However, the finitude of samples makes this a familiar pattern. In the case at hand, the variables were all bounded. GLWCD's dependent variable was transformed into a per-month measure, but was in fact a bounded six-point scale for the ROOM data, and a seven point scale for the Add Health data for the item "over the past year"; for the dependent variable "over the past two weeks" there was a count that can go from 0 to 14 (every day).

To demonstrate this for those who have not encountered this pattern before, this appendix presents simulated data. Here I present what happens when we have a strong predictor $\left(x_{1}\right)$ of a dichotomy, and we then look for the effects of a second predictor $\left(x_{2}\right)$ along bins formed by values of $x_{1}$, as do GLWCD. I present an example data, the results of 100 simulations, and then the R-code that can be used to replicate. Because of the difficulty of simulating data that fit both an OLS regression (which GLWCD used) and a bounded count variable, I here use a logistic regression for purposes of illustration.

The figure below shows simulated data, with a strong predictor on the horizontal axis, and a weak predictor on the vertical. Each dot is a data point, and the dot is colored red (or dark, if this is printed in black/white) if the dichotomous dependent variable is 0 , and green (or light) if it is 1. The equation is $y=b_{1} x_{1}+b_{2} x_{2}+\varepsilon$, where $b_{1}=10 ; b_{2}=5$, and $\varepsilon=N(0,2)$, where both $x_{1}$ and $x_{2}$ are draws of 300 points from a uniform distribution between -1 and 1 . A dichotomous variable is then constructed $z=1$ if $y>0$. A logistic regression of $z$ on $x_{2}$ is carried out in each of 10 bins formed by breaking up $x_{1}$ into 10 equally sized levels. The p-value for each bin is placed as a black square, so that you can determine the relation between the data in the bin and the p-value. (Bin lines are not drawn to avoid clutter, but you can visually group data points horizontally near to the square.) 


\section{example results}

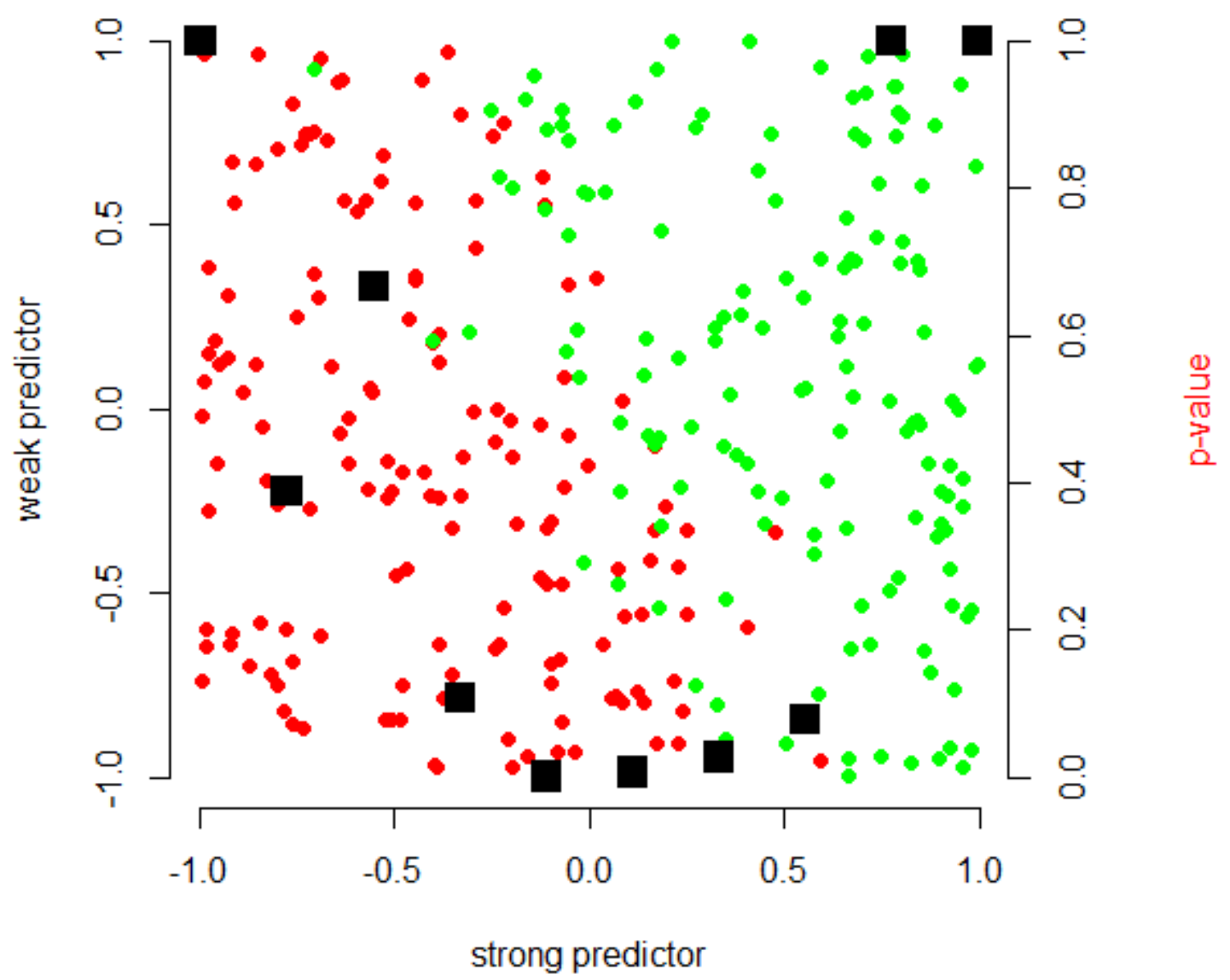


Doing this for 100 simulations, and averaging the p-values produces the following:

\section{average $p$-values}

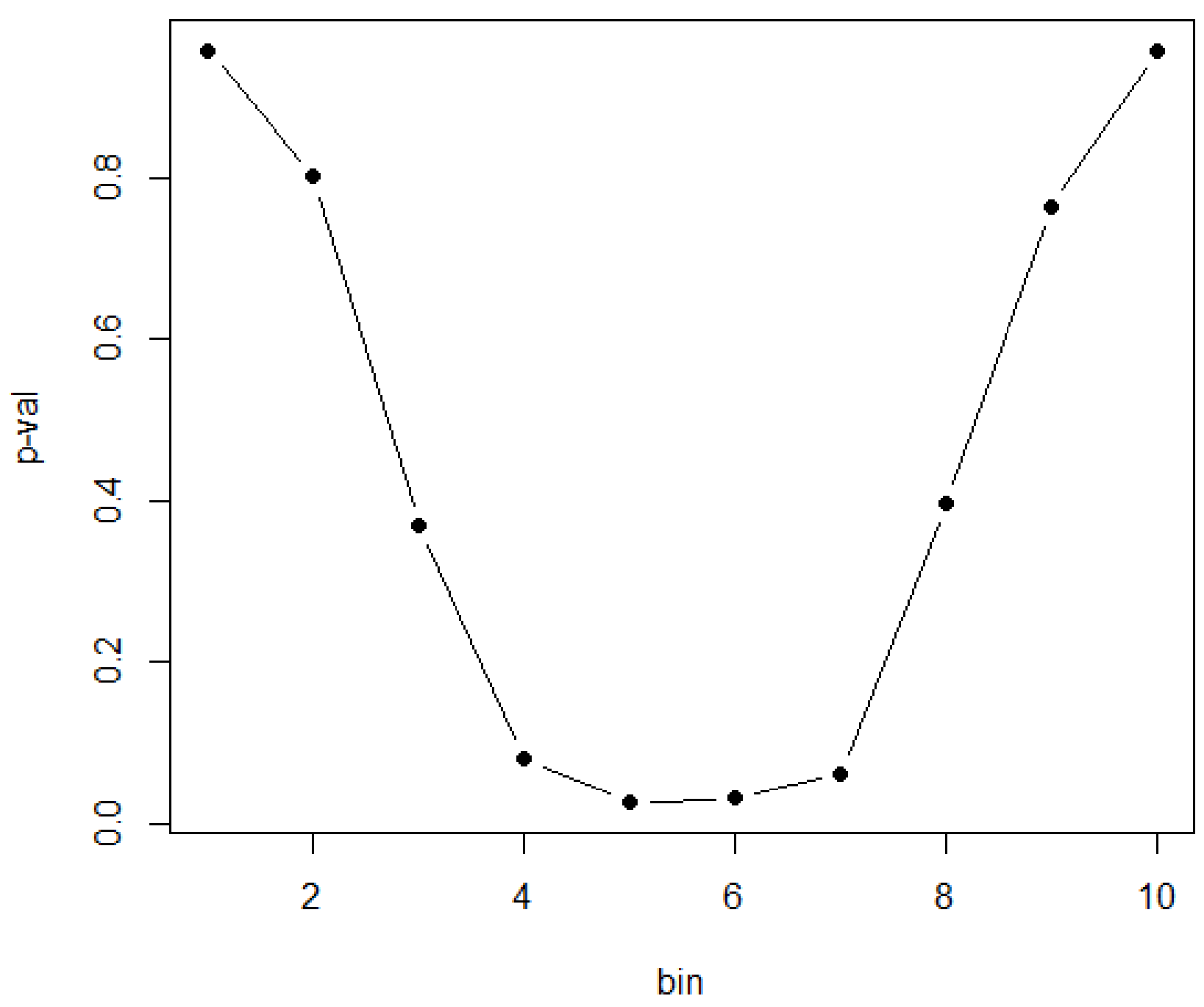

You will note the tendency for the weak predictor to only have explanatory power when the strong predictor is in the middle of its range. This is the case even though there is no interaction between the variables. The code below allows you to experiment with different values. The Rcode begins on the next page. 


\section{R-code}

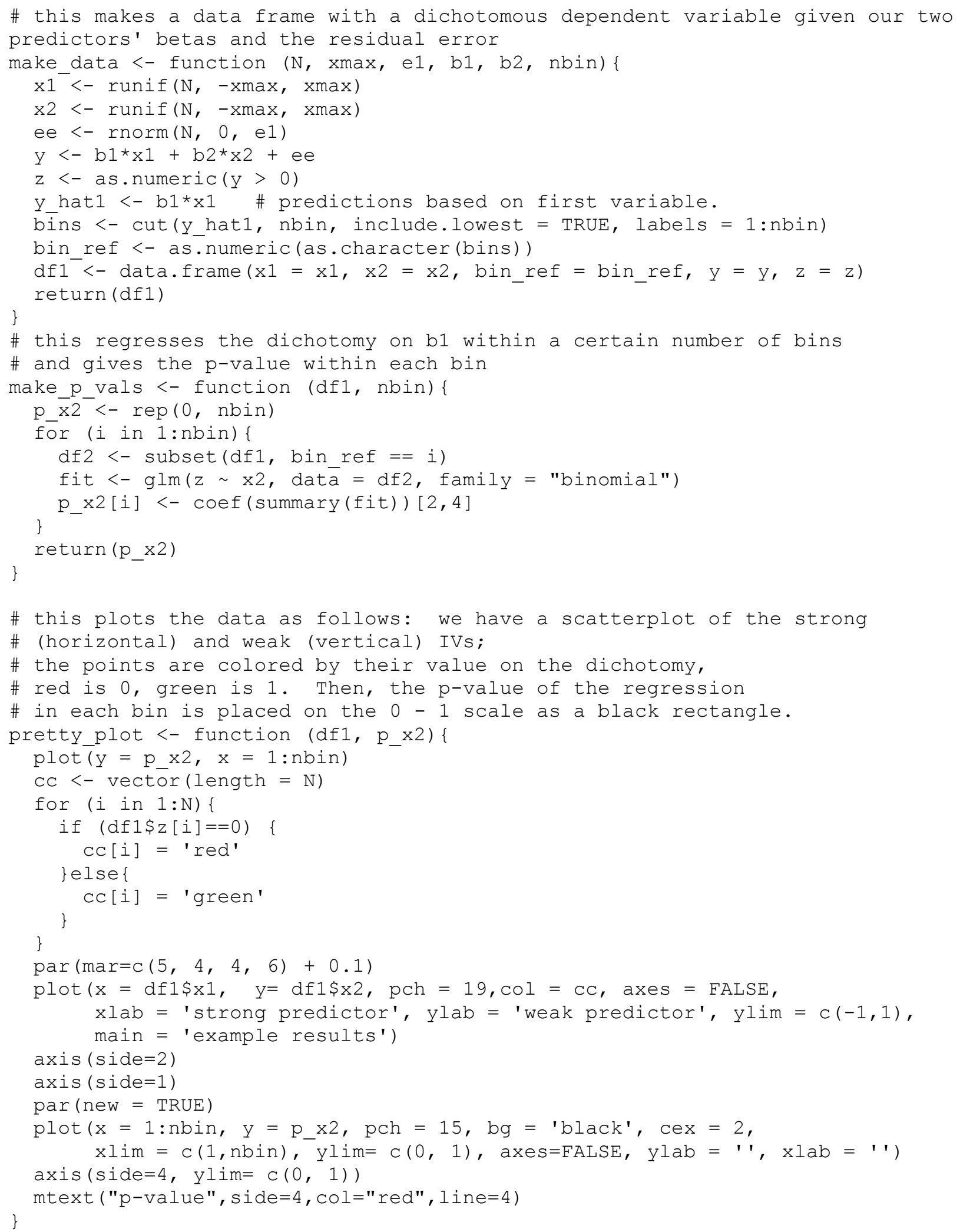




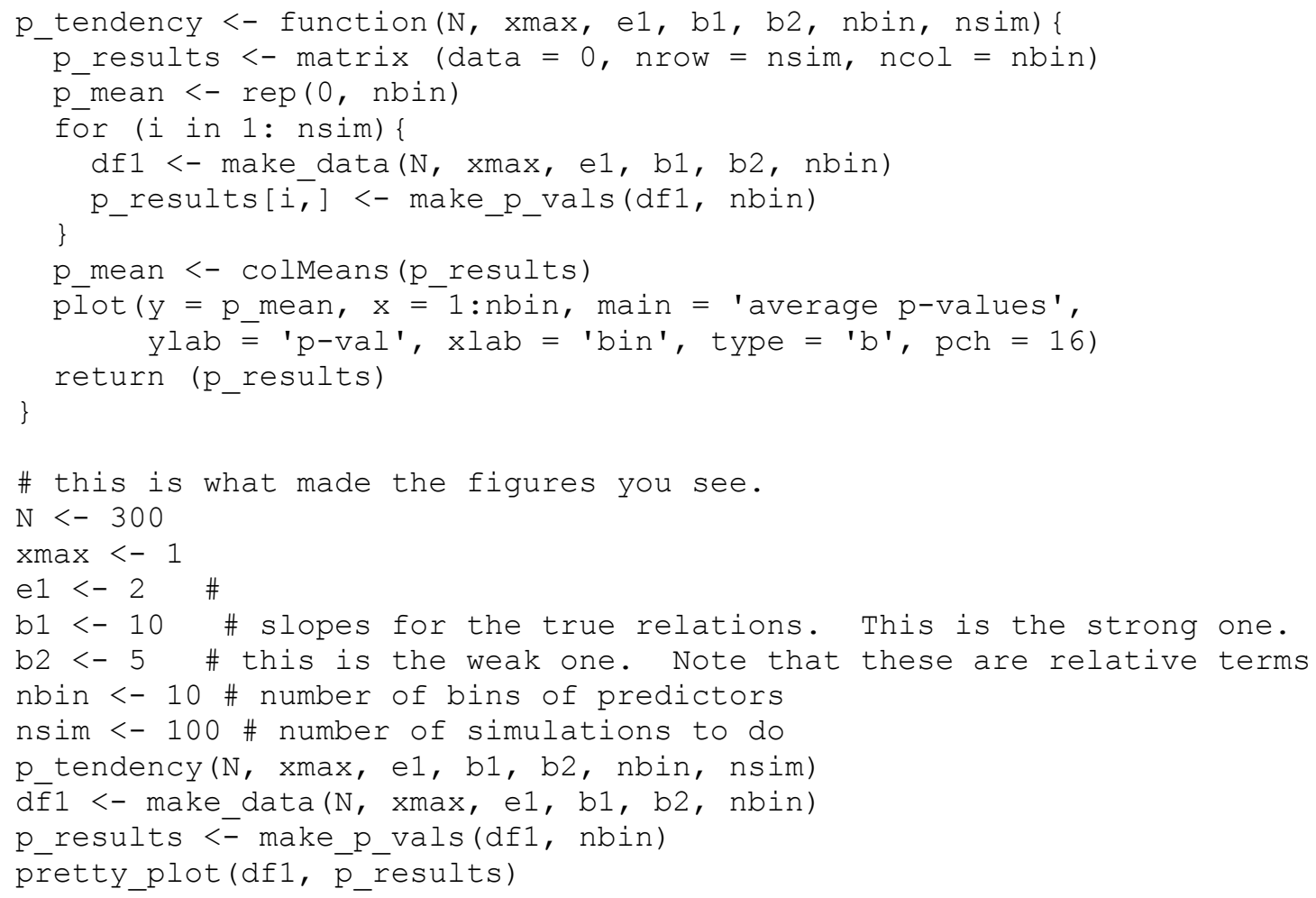


Appendix C: For those who doubt the claim that the specification of tiered models can lead to spurious interactions

I noted that GLWCD's method does not eliminate the possibility that the interaction of environment is with one of the many variables associated with genetic propensity, and not the propensity itself. This is true even if each individual regression includes these variables as controls. Here I demonstrate this for the simple case of a linear effect since this is relevant to the interpretation of GLWCD's findings as a more conventional gene $\times$ environment interaction-it is, however, a direct extension to the quadratic case in which the interaction is such that the effect of one variable is maximal in the middle of the range of another. Consider two correlated variables, $w$ and $z$, with a correlation of .5; here $z$ will represent a genetic propensity score and $w$ something like biodescent and/or socially ascribed race. Let $z$ not be a direct prediction of our outcome $y$, while $w$ is, and also interacts with a third variable, $x$, which might represent some environmental treatment. Thus the true equation is $y=b_{x} x+b_{w} w+b_{x w} x w+\varepsilon$, where $b_{x}=b_{w}=1$; $b_{x w}=2$, and $\varepsilon=N(0,1)$. When we have the interaction between the proper variables, we find that there is no main or interaction effect for $z$ (see Model 1, Table below), but we see such a spurious interaction if we omit the $x w$ interaction (Model 2).

\begin{tabular}{|l|l|l|}
\hline & Model 1 & Model 2 \\
\hline$z$ & -.015 & .161 \\
& $(.119)$ & $(.197)$ \\
\hline$w$ & $.885 * * *$ & $.742 * * *$ \\
& $(.055)$ & $(.092)$ \\
\hline$x$ & $.917 * * *$ & $.856 * *$ \\
& $(.094)$ & $(.157)$ \\
\hline$x z$ & -.027 & $2.222 * * *$ \\
& $(.203)$ & $(.296)$ \\
\hline$x w$ & $2.011 * * *$ & \\
& $(.088)$ & .392 \\
\hline$R^{2}$ & .784 & \\
\hline
\end{tabular}

(Here and below, $* * * \mathrm{p}<.001 ; * * \mathrm{p}<.01$.

We might imagine that this problem goes away if we split the data by $z$, as done by GLWCD. But this is not so. Here we break the data up into three equal parts by the value on $z$ and regress $y$ on $x$, controlling for $w$ :

\begin{tabular}{|l|c|c|c|}
\hline & \multicolumn{3}{|c|}{ Value on $z$} \\
\hline Coefficients & LOW & MEDIUM & HIGH \\
\hline$w$ & $.785 * * *$ & $.700^{* * *}$ & $.675^{* * *}$ \\
\hline$x$ & -.535 & $.682 * *$ & $2.466 * * *$ \\
\hline
\end{tabular}

We see that the value of the regression coefficient for $x$ varies dramatically across the categories of $z$, even though there is no interaction between $z$ and $x$, and we are controlling for $w$. Thus the presence of significant results at some but not all categories should only be taken as a rejection 
of a model that the groups formed by splitting on propensity are identical, and not that there is an interaction between propensity and the measured indicators.

$\mathrm{R}$-code allowing the reader to replicate begins on the next page.

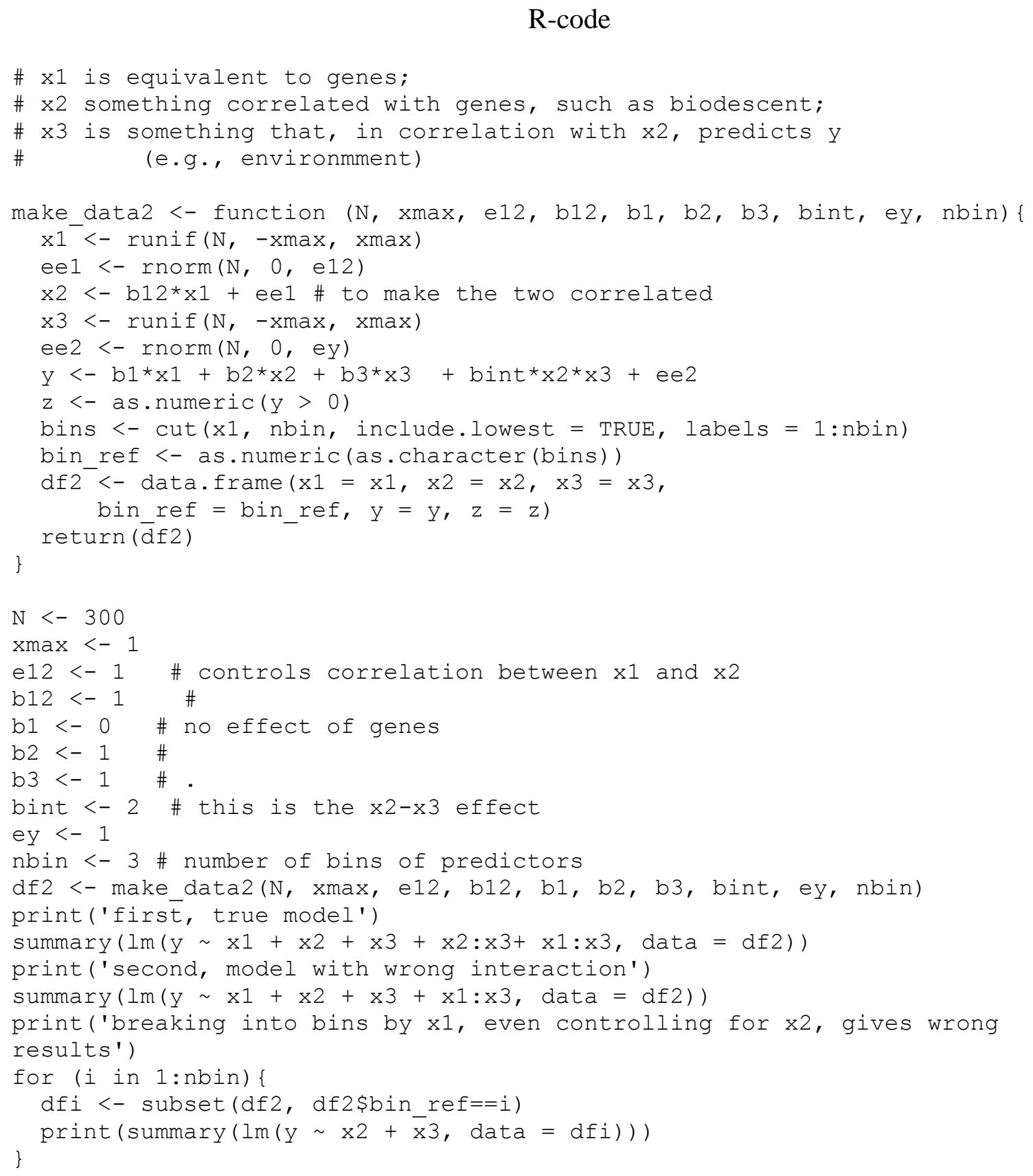

